Article

\title{
Iron and Acinetobacter baumannii Biofilm Formation
}

\author{
Valentina Gentile, Emanuela Frangipani, Carlo Bonchi, Fabrizia Minandri, \\ Federica Runci and Paolo Visca*
}

Department of Sciences, Roma Tre University, Viale Marconi 446, 00146 Rome, Italy; E-Mails: valentina.gentile@uniroma3.it (V.G.); emanuela.frangipani@uniroma3.it (E.F.); carlo.bonchi@uniroma3.it (C.B.); fabrizia.minandri@uniroma3.it (F.M.); federica.run@gmail.com (F.R.)

* Author to whom correspondence should be addressed; E-Mail: paolo.visca@uniroma3.it; Tel.: +39-06-57336347; Fax: +39-06-57336321.

Received: 10 July 2014; in revised form: 9 August 2014 / Accepted: 12 August 2014 / Published: 18 August 2014

\begin{abstract}
Acinetobacter baumannii is an emerging nosocomial pathogen, responsible for infection outbreaks worldwide. The pathogenicity of this bacterium is mainly due to its multidrug-resistance and ability to form biofilm on abiotic surfaces, which facilitate long-term persistence in the hospital setting. Given the crucial role of iron in A. baumannii nutrition and pathogenicity, iron metabolism has been considered as a possible target for chelation-based antibacterial chemotherapy. In this study, we investigated the effect of iron restriction on $A$. baumannii growth and biofilm formation using different iron chelators and culture conditions. We report substantial inter-strain variability and growth medium-dependence for biofilm formation by $A$. baumannii isolates from veterinary and clinical sources. Neither planktonic nor biofilm growth of $A$. baumannii was affected by exogenous chelators. Biofilm formation was either stimulated by iron or not responsive to iron in the majority of isolates tested, indicating that iron starvation is not sensed as an overall biofilm-inducing stimulus by $A$. baumannii. The impressive iron withholding capacity of this bacterium should be taken into account for future development of chelation-based antimicrobial and anti-biofilm therapies.
\end{abstract}

Keywords: Acinetobacter baumannii; biofilm; chelator; deferasirox; deferiprone; deferoxamine; dipyridyl; iron; transferrin 


\section{Introduction}

Acinetobacter baumannii has emerged worldwide as a leading cause of hospital-acquired infections, especially among severely ill patients in intensive care units (ICUs) [1]. Although A. baumannii was initially regarded to as a low-grade pathogen, evidence has been accumulated suggesting that A. baumannii infections are associated with increased mortality in critically ill patients [2]. A. baumannii causes a broad range of nosocomial infections, including ventilator-associated pneumonia, urinary tract infections, wound infection, bacteremia, endocarditis, meningitis [3], and has recently been associated with very severe community-acquired infections, especially among individuals with predisposing factors in Southern Asia and other tropical regions [4]. A. baumannii can also be isolated from veterinary sources, and show common characteristics with strains described in human infection [5].

Tendency to the epidemic spread, resistance to antibiotics and persistence in the hospital setting are hallmarks of $A$. baumannii infection [3]. Successful strains of multidrug-resistant (MDR) A. baumannii are notorious for their ability to rapidly spread among hospitalized patients, overcome geographical borders, and become epidemic worldwide [6]. Epidemiologic and population genetics studies indicate that the majority of $A$. baumannii infections are caused by strains belonging to three international clonal lineages (ICLs) [1,3,6]. A. baumannii strains belonging to the most widespread ICLs are invariably characterized by an MDR phenotype, which is progressively evolving towards pandrug resistance, thereby challenging the current antimicrobial armamentarium [7,8]. This poses the urgent need for the development of novel treatment strategies to combat infections caused by MDR A. baumannii [9].

The capacity of MDR clinical isolates of $A$. baumannii to resist to desiccation and to form biofilms are regarded as crucial factors contributing to the clinical success and persistence of this species in healthcare facilities. A. baumannii can survive for up to months on the dry surface of inanimate objects [10,11], enabling transmission of infection for long times under both epidemic and endemic situations [12]. A number of reports have demonstrated that A. baumannii can form biofilms on several biotic and abiotic surfaces, providing the bacteria with protection against antibiotic/antiseptic treatment(s) and the host immune defenses in vivo (reviewed in $[13,14]$ ). Biofilm formation is crucial for several A. baumannii infections, since these are often associated with indwelling medical devices, e.g., vascular and urinary catheters, cerebrospinal fluid shunts, and endotracheal tubes [15]. While it is apparent that the capacity to form biofilms is a general phenotypic trait of $A$. baumannii, remarkable differences in the amount of biofilm formed by different strains have been reported, even if belonging to the same clonal lineage or epidemiological cluster [12,15-18]. A number of environmental factors can influence biofilm formation, including the presence of metal cations [16,19]. Among these, iron represents an essential nutrient for infecting bacteria, and a key determinant in host-pathogen interactions. This is because bacteria must counteract an iron-poor environment during infection, due to iron sequestration by iron carrier and storage proteins of the host and adaptive hypoferremia during infection [20]. A. baumannii has evolved an impressive capacity to acquire iron from the host, due to the production of multiple siderophores for Fe(III) transport, combined with uptake specificities for heme and $\mathrm{Fe}(\mathrm{II})[21,22]$.

Given the crucial role of iron in A. baumannii-host interactions [22-24], attention has recently been given to non-antibiotic approaches that target iron metabolism to achieve antibacterial activity, 
including chelation therapy and use of iron mimetics (reviewed in [9]). Interestingly, it was noted that: (i) high concentrations of deferiprone (DFP, (Sigma Aldrich, St. Louis, MO, USA)), a compound used for chelation therapy in humans, inhibited to some extent logarithmic growth of A. baumannii ATCC 17978 in a chemically defined medium [25]; (ii) gallium, an iron-mimetic drug, suppressed the growth of MDR A. baumannii strains both in vitro and in vivo, acting through disruption of bacterial iron metabolism [25-27]; (iii) mutants impaired in production of the acinetobactin siderophore show reduced fitness in vivo [23]. On the other hand, it was also reported that biofilm formation on plastic by the type strain A. baumannii ATCC $19606^{\mathrm{T}}$ was stimulated under conditions of iron scarcity imposed by the addition of the chelator 2,2'-dipyridyl (DIP) [19]. Therefore, the effect of iron availability on both planktonic and biofilm mode of $A$. baumannii growth deserves more in-depth investigation.

In this report, strains and optimal growth conditions for the generation A. baumannii biofilms were preliminarily established. Then, the role of iron in A. baumannii biofilm formation was investigated. Lastly, the activity of a new therapeutic iron chelator was assessed in search for inhibitory drugs that could be repurposed as adjuvant antimicrobials in the treatment of biofilm-based A. baumannii infections.

\section{Results and Discussion}

\subsection{Definition of Culture Conditions for A. baumannii Biofilm Formation}

Biofilm formation is a multifactorial phenotype [13,14], and in A. baumannii it can be modulated by iron availability, carbon sources, growth temperature, and different expression levels of adhesive and cell-aggregating factors $[13,14,16,18,19,28]$. Therefore, as a preliminary step to the investigation of the effect of iron on $A$. baumannii biofilm formation, we determined the growth response of the reference strain A. baumannii ATCC 17978 [25,29] to iron restriction imposed by different chelators in M9 minimal medium [30] containing $20 \mathrm{mM}$ sodium succinate as the carbon source [26]. In line with previous observations [25], A. baumannii ATCC 17978 showed an impressive ability to multiply under conditions of iron deficiency, as those imposed by the addition of up to $128 \mu \mathrm{M}$ human apo-transferrin (h-TF (Sigma Aldrich)), trisodium citrate (CIT (Sigma Aldrich)), desferrioxamine (DFO (Ciba Geigy, Origgio, Italy)), deferasirox (DFX (Novartis, Basel, Switzerland)) and DFP (Figure 1).

None of the tested chelators reduced A. baumannii ATCC 17978 growth yields at $48 \mathrm{~h}$, even when $100 \mu \mathrm{M}$ DIP (a chelator of the intracellular Fe(II) pool) was added to further reduce iron availability. As expected, growth in M9 was stimulated by $c a$. $25 \%$ in the presence of $100 \mu \mathrm{M} \mathrm{FeCl}_{3}$. A similar resistance to exogenously supplied chelators in M9 was also observed for strains AYE [31] and ACICU [32], representatives for ICL-I and ICL-II, respectively (data not shown). These data can be explained by the presence in A. baumannii of very efficient iron uptake systems [11,21], capable of counteracting the iron withholding capacity of exogenously added chelators. The observation that DFX, DFO, and DFP do not stimulate bacterial growth in the presence of DIP (a chelator of the intracellular $\mathrm{Fe}(\mathrm{II})$ pool) suggests that these chelators are unlikely to serve as an iron source for A. baumannii. 
Figure 1. Effect of different iron chelators on planktonic growth of $A$. baumannii ATCC 17978. Bacteria were grown for $48 \mathrm{~h}$ at $37{ }^{\circ} \mathrm{C}$ in 96-wells microtiter plates containing $100 \mu \mathrm{L}$ M9 supplemented with the indicated iron chelator at different concentrations: $32 \mu \mathrm{M}$ (light grey bars), $128 \mu \mathrm{M}$ (dark grey bars) or $128 \mu \mathrm{M}$ chelator $+100 \mu \mathrm{M}$ DIP (black bars). Growth was measured as $\mathrm{OD}_{600}$ and expressed as percentage relative to the untreated control (i.e., $\mathrm{OD}_{600}$ in M9). The average of the $\mathrm{OD}_{600}$ in control M9 was $0.318 \pm 0.008$ and represents $100 \%$ of growth (white bar). Relative growth in M9 supplemented with $100 \mu \mathrm{M}$ $\mathrm{FeCl}_{3}$ is reported (striped bar). Data represent the average of three independent experiments \pm standard deviation. h-TF, tranferrin; CIT, citrate; DFX, deferasirox; DFO, desferrioxamine; DFP, deferiprone.

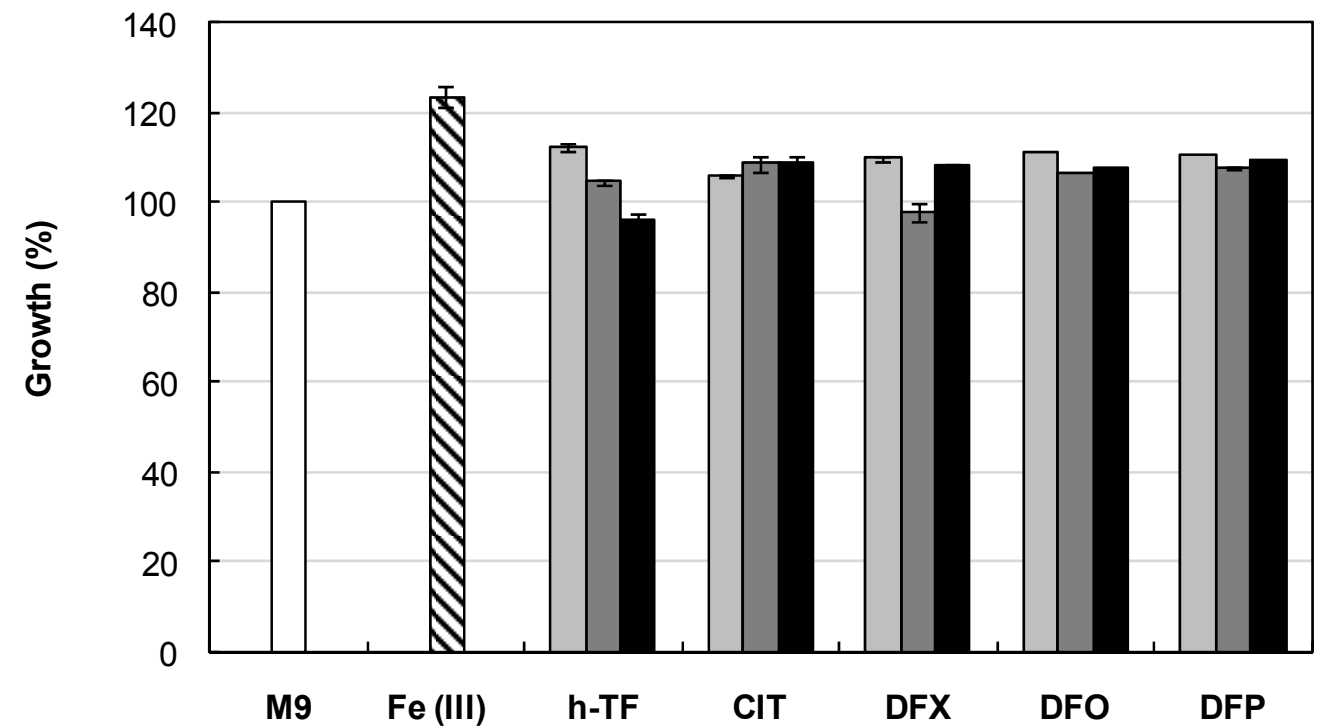

Next, the inter-strain variability and the growth medium-dependence of biofilm formation was investigated. Five well-characterized A. baumannii strains (AYE, representative for ICL-I [31]; ACICU, representative for ICL-II [32]; 50C, ICL-II pandrug resistant isolate [33]; RUH5875, prototypic strain for ICL-III [34]; ATCC 17978 [29]] were grown in three iron-poor media [M9, M9 supplemented with $100 \mu \mathrm{M}$ DIP, and Chelex-100-treated Tryptic Soy Broth dialysate, TSBD [35]) in order to determine both growth and biofilm levels at 24 and $48 \mathrm{~h}$. Quantitative estimation of the bacterial biomass in biofilms was assessed in 96-well polystyrene microtiter plates (BD Falcon, Milano, Italy), using the crystal-violet (CV) staining method [36]. There was a wide range of variation in growth and biofilm levels between $A$. baumannii, depending on strains and culture media, though for some strains moderate correlation was observed between growth yields and biofilm levels (Figure 2).

Remarkably, all strains produced more abundant biofilm in TSBD than in the other iron-poor media, and biofilm levels in strain ACICU were significantly higher ( $p<0.05$ in the student's $t$-test) than all the other strains tested (Figure 2). These findings corroborate the notion that biofilm levels in A. baumannii can vary even between closely related isolates (e.g., strains ACICU and 50C belonging to the same genetic cluster according to ref. [33]), and that different media have a profound impact on biofilm yields [15-18].

To rule out the possibility that differences in biofilm levels between TSBD and M9 or M9 plus DIP were due to different iron content of these media, an iron biosensor consisting of the Fur-controlled 
bas $A$ promoter fused to the reporter lacZ gene [26] was used as a probe to determine the intracellular iron level in A. baumannii ATCC17978. Since the Fur repressor protein acts as an iron sensor, the activity of the Fur-controlled bas $A$ promoter provides an indirect estimate of the intracellular iron levels of bacteria grown in the different media. The $\beta$-galactosidase (LacZ) expression was higher in TSBD than in M9 or M9 plus DIP (Figure 3), and it was invariably repressed by iron, indicating that TSBD is sensed by $A$. baumannii as an iron-poor medium.

Figure 2. Growth and biofilm formation by selected $A$. baumannii strains in different iron-poor media. Bacterial cells were inoculated at $\mathrm{OD}_{600}$ of 0.01 in $100 \mu \mathrm{L}$ of the different growth media, dispensed in a 96-wells microtiter plate, and grown at $37{ }^{\circ} \mathrm{C}$ without shaking for 24 and $48 \mathrm{~h}$. Growth (circles) was measured spectrophotometrically $\left(\mathrm{OD}_{600}\right)$ and biofilm formation (bars) was evaluated using the CV staining assay [36]. Dark grey, TSBD; light grey M9; white M9 supplemented with $100 \mu \mathrm{M}$ DIP. Data represent the average of three independent experiments \pm standard deviation.

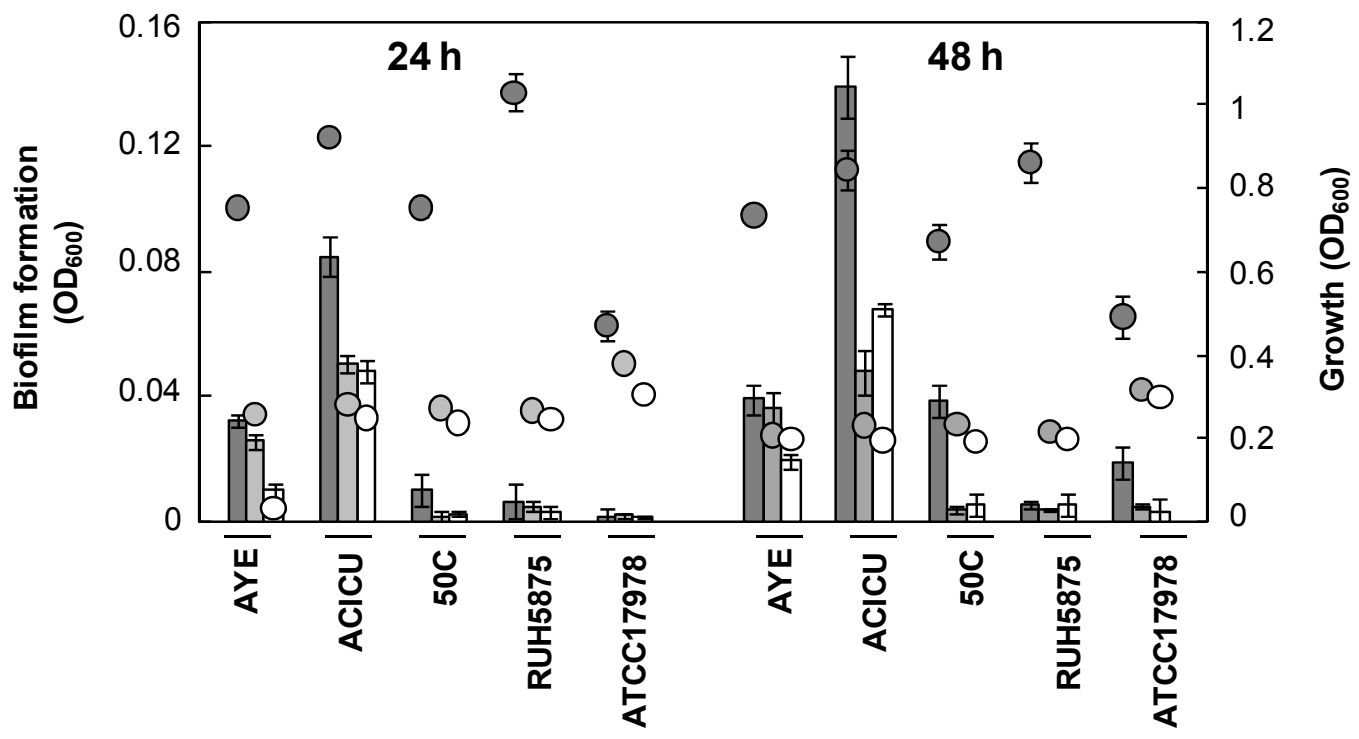

To visualize differences in biofilm structure among the five representative A. baumannii strains, biofilm formation on glass slides was monitored during seven days growth in TSBD by means of confocal microscopy, according to a previously described procedure [37] (Figure 4). High biofilm levels with formation of large cellular aggregates were observed for $A$. baumannii ACICU, and to a much lesser extent for the other strains (Figure 4A). Interestingly, A. baumannii biofilm cells were found to be embedded in a blue fluorescent material upon staining with calcofluor white (Figure 4B). In line with previous findings [18,29], this observation denotes the presence of exopolysaccharides in the matrix of $A$. baumannii biofilms, whose levels appear to be consistent with to the amount of biofilm formed in 96-well polystyrene microtiter plates (Figure 2).

Based on the above results, TSBD was considered as suitable iron-depleted medium that would allow robust biofilm formation and an easier evaluation of the effect of iron on this process. This is because the high biofilm levels achieved by A. baumannii in TSBD would facilitate the detection of 
biofilm variations in response to iron levels. Moreover, the high peptide content and balanced formula of TSBD (35, see also [38]) make it more similar to a biological fluid than the M9 mineral salt medium.

Figure 3. Regulatory mechanism and activity of the basA::lacZ iron biosensor in the reference A. baumannii strain ATCC 17978. (A) Schematic of the regulatory mechanism the bas $A:: l a c Z$ iron-regulated transcriptional fusion carried by plasmid pMP220:: $\mathrm{P}_{\text {basA }}$ [26]. Under iron proficient conditions (left), the Fur repressor protein binds the $\mathrm{P}_{\text {bas }}$ promoter and inhibits $\beta$-galactosidase (LacZ) expression; under iron deficient conditions Fur repression is relieved and the LacZ enzyme is expressed. (B) Activity of the basA::lacZ iron-regulated fusion in A. baumannii ATCC 17978 grown for 24 and $48 \mathrm{~h}$ in different media, as indicated, in the absence (white bars) or presence (black bars) of $100 \mu \mathrm{M} \mathrm{FeCl}$. Data are the means $( \pm$ standard deviations $(\mathrm{SD}))$ of triplicate experiments.

A

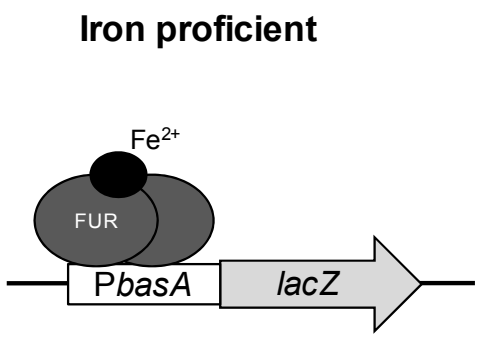

lacZ repression
Iron deficient

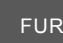

FUR

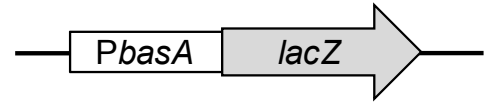

lacZ expression

B

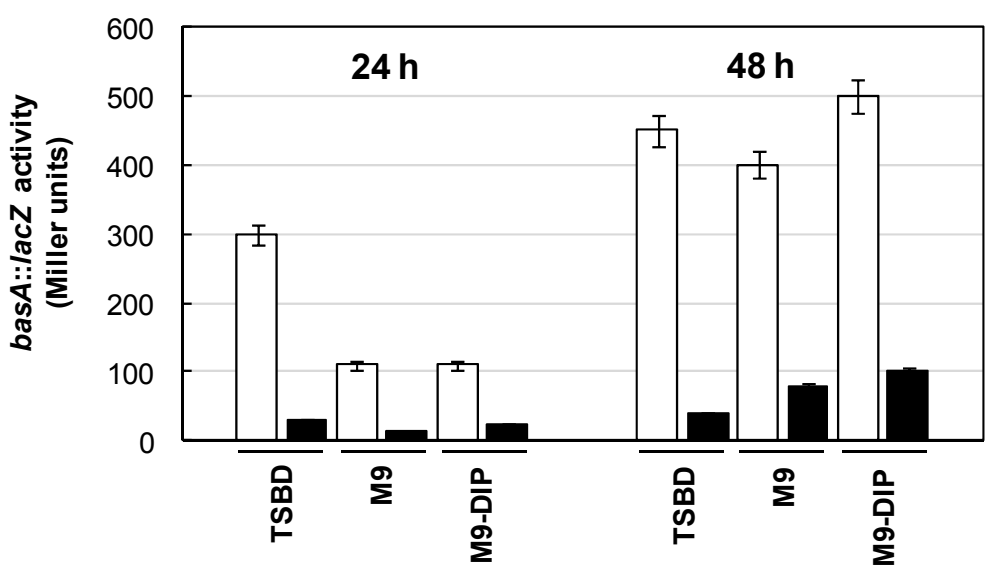


Figure 4. Seven-days biofilm of selected A. baumannii strains grown in TSBD. (A) Confocal microscope images (x-y plane and side view) of A. baumannii biofilms stained with acridine orange, a fluorescent dye which labels double-stranded nucleic acids (prevalently DNA) in green, and single-stranded nucleic acids (prevalently RNA) in red. (B) A. baumannii biofilms stained with the calcofluor white for exopolysaccharide labelling [19,28], and analyzed by fluorescence microscopy. Scale bar: $50 \mu \mathrm{m}$.

\section{A}
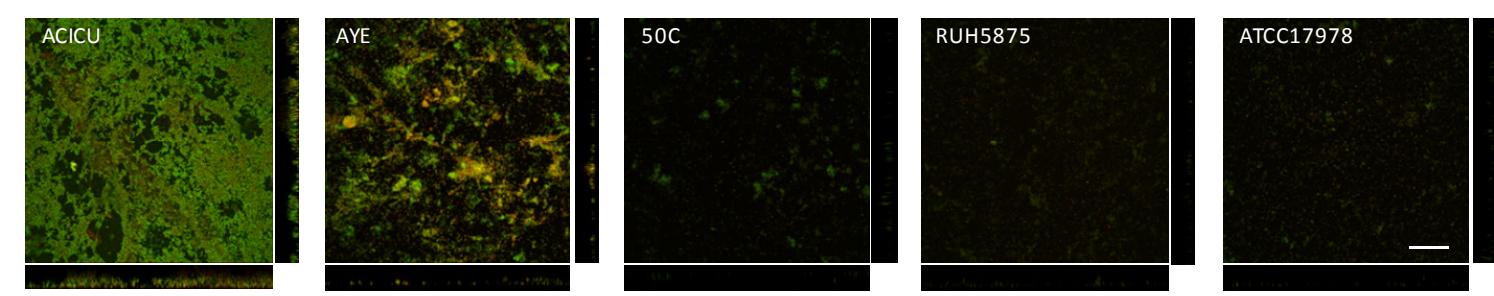

B
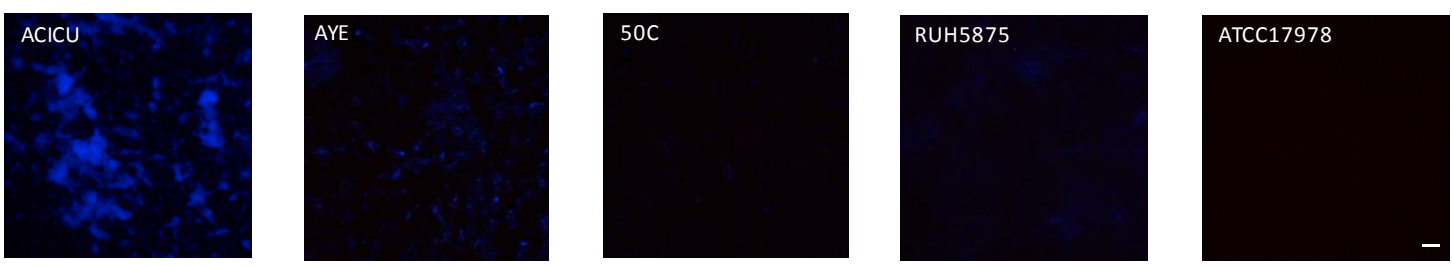

\subsection{Effect of Iron Levels on Biofilm Formation by a Collection of Diverse A. baumannii Isolates}

We investigated the effect of iron on biofilm formation in a representative collection of 54 A. baumannii strains (Table S1 in Supplementary Material), including 27 isolates from veterinary sources (67\% MDR) and 27 isolates from clinical sources (96\% MDR). Isolates were selected so to maximize diversity, as inferred by Random Amplified Polymorphic DNA fingerprints with primer DAF4 ([21,33]; data not shown), and represent the most widespread ICLs and some emerging lineages (Table S1 in Supplementary Material). High growth yields were observed for almost all isolates in TSBD (median $\mathrm{OD}_{600}=0.716$ ), which were significantly increased by the addition of $100 \mu \mathrm{M} \mathrm{FeCl}_{3}$ (median $\mathrm{OD}_{600}=1.031$ ), consistent with iron being a nutritionally-limiting factor in TSBD (Figure 5A). Remarkably, biofilm formation was more abundant in $\mathrm{FeCl}_{3}$-supplemented TSBD (median $\mathrm{OD}_{600}=0.102$ ) than in TSBD (median $\mathrm{OD}_{600}=0.071$ ) (Figure 5B). After having excluded from the analysis 4 biofilm non-producing isolates (namely, 4297, 196-1, 82D, RUH5875, see Table S1), the normalization of biofilm formation by growth yields resulted in minor differences between the two growth conditions (median values were 0.104 and 0.098 for the iron-limited and iron-rich condition, Figure 5C). This result is due to somehow opposite responses of A. baumannii isolates to iron starvation (i.e., TSBD vs. $\mathrm{FeCl}_{3}$-supplemented TSBD); in 21 isolates (42\%) biofilm production was significantly enhanced by iron deficiency, in 12 (24\%) it was significantly reduced, and in 17 (34\%) iron had no effect on biofilm formation (significance in the Student's $t$-test was set at $p<0.05$ ). Although stimulation of biofilm formation in response to iron-limited growth was observed for a minority (42\%) of $A$. baumannii isolates, this may have relevant medical implications, since transition of these isolates from planktonic to biofilm-growing cells could be favored in vivo, where infecting bacteria are normally challenged with iron shortage. However, this behavior cannot be generalized, 
since biofilm production was either unchanged or even inhibited by iron deficiency in the majority $(66 \%)$ of the isolates.

\subsection{Effect of Deferasirox on A. baumannii Biofilm Formation}

We showed that planktonic $A$. baumannii has an impressive ability to grow in the presence of exogenously added therapeutic chelators DFP, DFO, and DFX (Figure 1), and previous data indicate only modest inhibition of $A$. baumannii growth at high DFP concentrations ( $c a .200 \mu \mathrm{M}$, ref. [25]). To gain further insight into the effect of iron withholding on A. baumannii biofilm formation, we examined the effect of DFX, a newly developed orally active Fe(III) chelator, on our collection of 50 biofilm-producing isolates. DFX is a synthetic compound with high affinity and specificity for $\mathrm{Fe}(\mathrm{III})\left(\log \beta_{2}=36.9\right.$ according to ref. [39]), and is unlikely to serve as an iron carrier to A. baumannii based on growth assays (Figure 1). It was successfully used in combination therapy against murine staphylococcemia [40], and in treatment of invasive fungal infections [41].

Here, biofilm formation was tested in a DFX concentration range 4-128 $\mu \mathrm{M}$, in order to match the DFX plasma levels achievable during treatment of iron overload in humans [42]. Notably, biofilm formation by most of the isolates was not significantly affected by DFX up to $128 \mu \mathrm{M}$, either in TSBD or in TSBD plus $100 \mu \mathrm{M} \mathrm{FeCl}_{3}$ (Figure 6A).

Figure 5. Growth and biofilm formation in a representative collection of 54 A. baumannii strains from clinical and veterinary sources. (A) Growth of 54 A. baumanni strains for $48 \mathrm{~h}$ in 96-wells microtiter plates containing $100 \mu \mathrm{L}$ TSBD supplemented (black circles) or not (white circles) with $100 \mu \mathrm{M} \mathrm{FeCl}_{3}$, as indicated. (B) Absolute values of biofilm formation by the same isolates shown in panel $A$, evaluated by the $\mathrm{CV}$ staining assay $\left(\mathrm{OD}_{600}\right)$. Grey circles (B) represent the values for strains that in either or both conditions yielded negative biofilm values, and were excluded from calculations in panel C. (C) Relative values of biofilm formation (Biofilm formation $\left(\mathrm{OD}_{600}\right)$ /Growth $\left(\mathrm{OD}_{600}\right)$ ) for a subset of 50 biofilm-producing isolates. The line bar represents the median value for each group. Values for each strain are the average of three independent experiments.

A

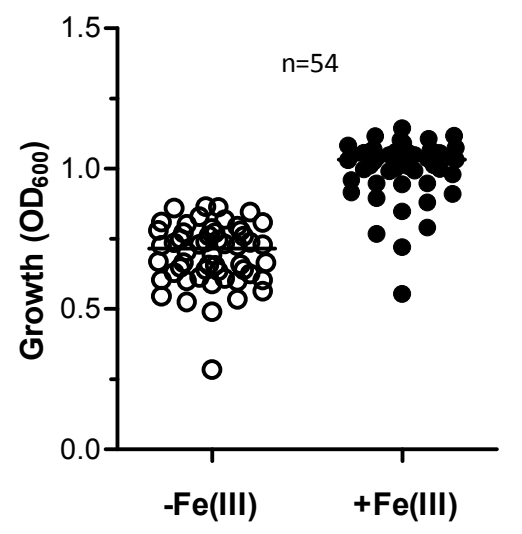

B

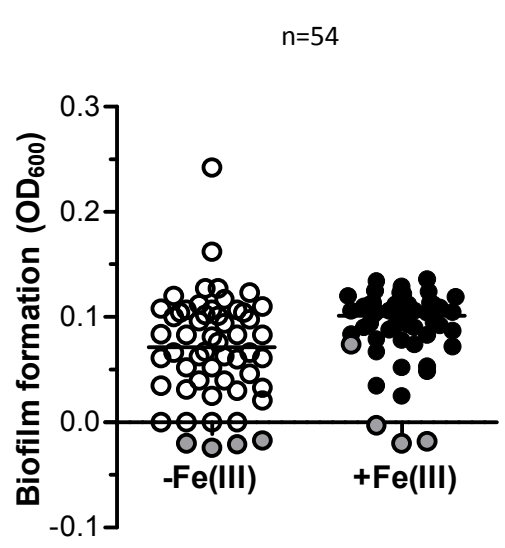

C

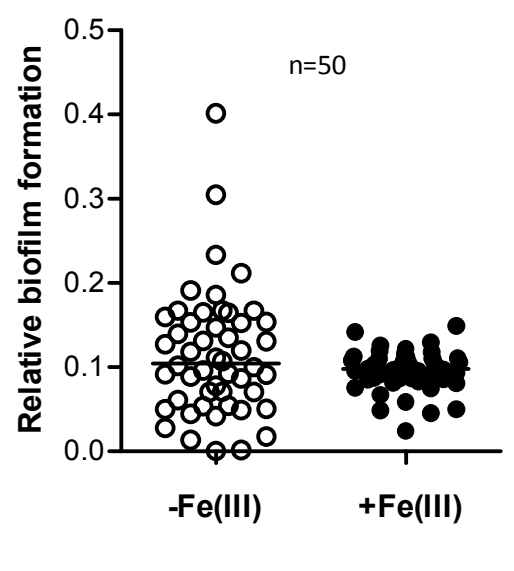


Figure 6. Effect of DFX on A. baumannii biofilm formation. (A) A. baumannii strains were grown statically for $48 \mathrm{~h}$ in microtiter plates containing $100 \mu \mathrm{L}$ TSBD supplemented with DFX at indicated concentrations, or $128 \mu \mathrm{M}$ DFX plus $100 \mu \mathrm{M} \mathrm{FeCl}$. Biofilm formation $\left(\mathrm{OD}_{600}\right.$ in the CV staining assay) was normalized by the growth yield $\left(\mathrm{OD}_{600}\right.$ of the culture) and expressed as percentage relative to the DFX-untreated control (TSBD). Boxes represent medians, second and third interquartiles; whiskers represent range of 50 isolates tested. (B) Relative biofilm levels produced by individual isolates in presence of $128 \mu \mathrm{M}$ DFX, expressed as \% of the untreated control in TSBD. With reference to Figure $5 \mathrm{C}$, the bar filling denotes: isolates in which biofilm production was significantly enhanced by iron deficiency (white, 21 isolates), or significantly reduced (black, 12 isolates), or in which iron had no effect on biofilm formation (grey, 17 isolates). In both panels data represent the average of three independent experiments.

\section{A}

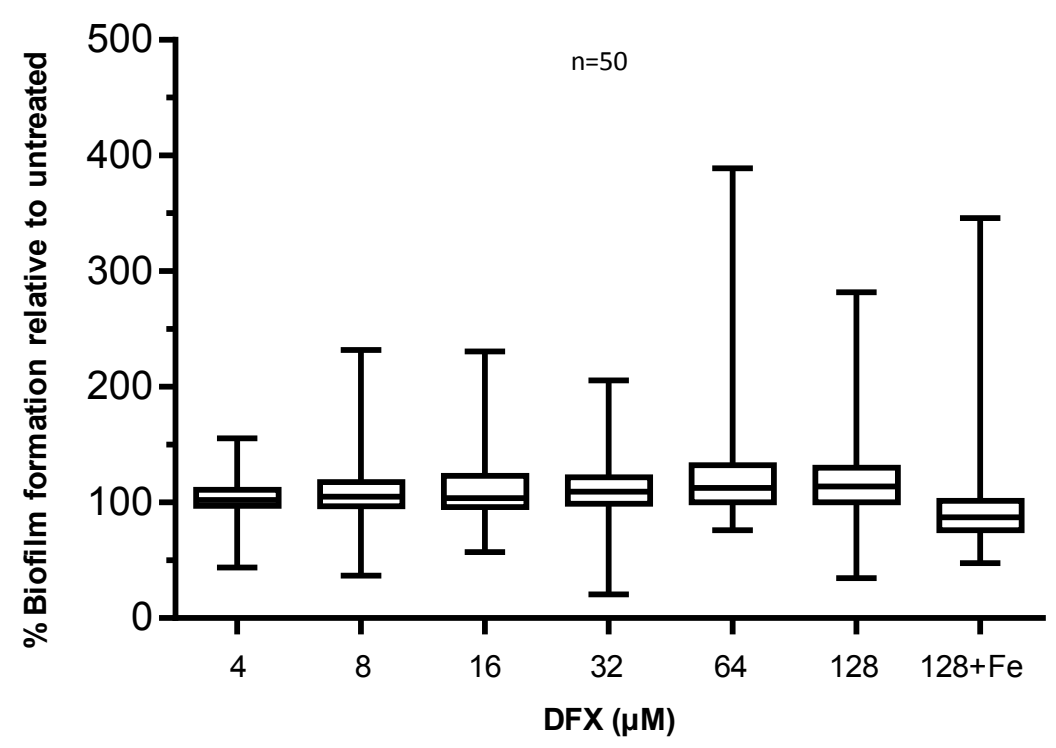

B

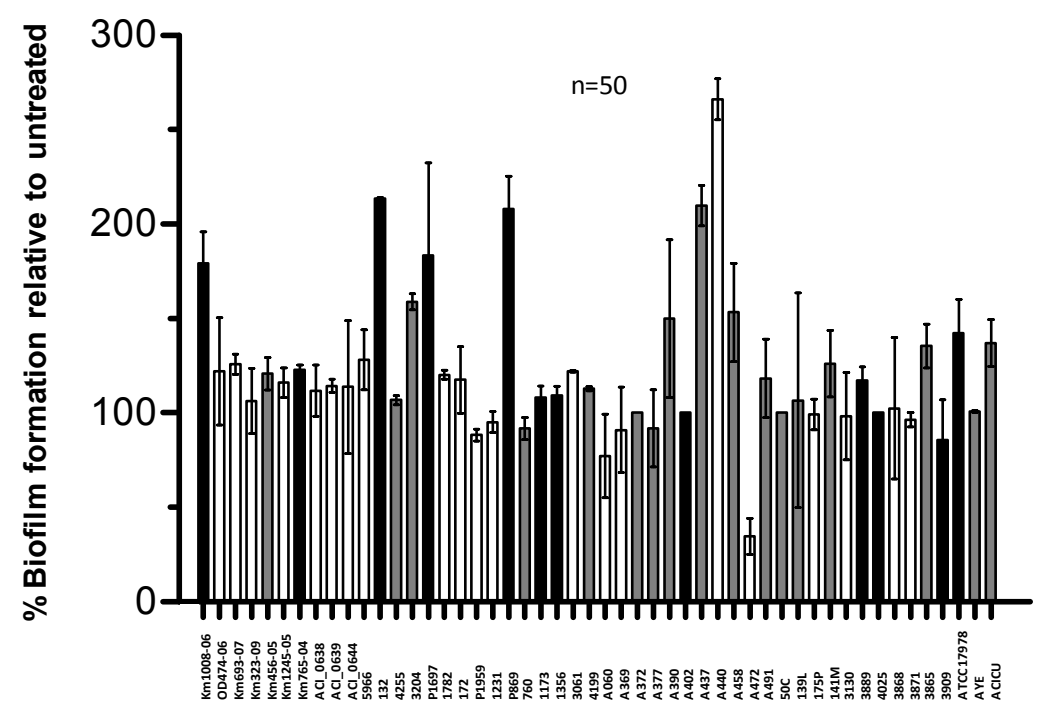


No obvious correlation could be observed between the biofilm response to iron starvation in TSBD (see Figure 5 and associated text) and in the presence of $128 \mu \mathrm{M}$ DFX (Figure 6B). The observation that a minority of isolates (i.e., Km1008-06, 132, P1697, P869, A472, and ATCC17978) showed opposite responses to iron starvation induced by TSBD with and without iron (Figure 5), compared with TSBD and TSBD plus DFX (Figure 6B), suggests that either DFX exerts iron chelation-independent effects, or that the iron deficiency threshold that determines the biofilm response in A. baumannii can vary for these isolates. Apart from this, the ability to generate biofilms is scarcely influenced by the presence of DFX for most $A$. baumannii isolates, indicating that, at least in vitro, this therapeutic chelator is incapable of overcoming the iron withholding capacity of A. baumannii biofilms.

\section{Experimental Section}

\subsection{Bacterial Strains and Growth Media}

Relevant characteristics of the $54 \mathrm{~A}$. baumannii strains used in this study are provided in Table S1 in the Supplementary Material. The collection includes representative strains for ICLs I, II, and III, namely AYE (ICL-I) [43], ACICU (ICL-II) [32], and RUH 5875 (ICL-III). Strain ATCC 17978 is a well-characterized clinical isolate dated 1950s and showing moderate antibiotic resistance [29]. Strain $50 \mathrm{C}$ is a pandrug resistant clinical isolate $[33,44]$. Other clinical and veterinary isolates were provided as part of the collections from various European laboratories [5,21,45-48]. Iron-poor culture media used in this study were the M9 minimal medium [30] supplemented with $20 \mathrm{mM}$ sodium succinate as the carbon source, and TSBD, a Chelex 100-treated Trypticase Soy Broth dialysate [35]. When required, media were supplemented with either $100 \mu \mathrm{M}$ DIP or $100 \mu \mathrm{M} \mathrm{FeCl}_{3}$.

\subsection{Chemicals}

The chemicals used in this study were deferiprone (DFP, (Sigma Aldrich)), tri-sodium citrate [CIT, (Sigma Aldrich), desferrioxamine (DFO, (Ciba Geigy)); human apo-transferrin (h-TF, iron content $\leq$ 0.005\%, (Sigma Aldrich)), and deferasirox (DFX, (Novartis)).

\subsection{Growth Inhibitory Activity of Iron-Chelators}

The activity of the iron chelators on bacterial growth was tested in 96-well microtiter plates (BD Falcon) containing increasing concentrations $(4-128 \mu \mathrm{M})$ of iron chelators. Plates were inoculated at $\mathrm{OD}_{600}$ of 0.01 in a $100 \mu \mathrm{L}$ final volume of M9 supplemented or not with $100 \mu \mathrm{M}$ DIP at the highest iron-chelator concentration tested, or $100 \mu \mathrm{M} \mathrm{FeCl}_{3}$, and incubated at $37^{\circ} \mathrm{C}$ for $48 \mathrm{~h}$ with moderate shaking (100 r.p.m.). Spectrophotometric readings were performed in a Wallac 1420 Victor3V multilabel plate reader (Perkin Elmer, Milano, Italy).

\subsection{Biofilm Formation}

Biofilm formation was measured according to the microtiter plate assay [36]. Briefly, bacterial cells were inoculated at $\mathrm{OD}_{600}$ of 0.01 in $100 \mu \mathrm{L}$ of medium and grown at $37{ }^{\circ} \mathrm{C}$ for up to $48 \mathrm{~h}$ in 96 -wells 
microtiter plates without shaking. Planktonic cells were removed and the attached cells were gently washed three times with sterile PBS, air dried, and stained with $150 \mu \mathrm{L}$ of $0.1 \% \mathrm{CV}$ water solution for $20 \mathrm{~min}$. The wells were gently washed four times with distilled water, and the surface-associated dye was eluted in $200 \mu \mathrm{L}$ of $95 \%$ ethanol. The $\mathrm{OD}_{600}$ of the eluate was measured in a Wallac 1420 Victor3V multilabel plate reader (Perkin Elmer).

\subsection{Biofilm Inhibition}

To investigate the effect of DFX on biofilm formation, fifty biofilm-producing A. baumannii strains were inoculated $\left(\mathrm{OD}_{600}\right.$ of 0.01$)$ into 96-well microtiter plates containing $100 \mu \mathrm{L}$ TSBD supplemented with increasing DFX concentrations (4 to $128 \mu \mathrm{M}$ ) or $128 \mu \mathrm{M}$ DFX plus $100 \mu \mathrm{M} \mathrm{FeCl}_{3}$ The assay was performed as described above.

\subsection{Microscopy Analysis}

For microscopic visualization of $A$. baumannii biofilms, strains were grown in an 8-well chamber slide as previously described [37]. Briefly, bacteria were inoculated at $\mathrm{OD}_{600}$ of 0.01 in $200 \mu \mathrm{L}$ of TSBD and incubated at $37^{\circ} \mathrm{C}$ for $48 \mathrm{~h}$ to allow the adhesion of the bacterial cells on the glass surface. To maintain bacterial viability, the medium was changed every $24 \mathrm{~h}$ until the seventh day. To visualize biofilms structure, $A$. baumannii biofilms were stained with the acridine orange $(0.1 \%$ water solution), a fluorescent dye, which labels double-stranded nucleic acids (prevalently DNA) in green, and single-stranded nucleic acids (prevalently RNA) in red, and examined using Leica TCS SP5 confocal microscope. For detection of matrix exopolysaccharides, samples were stained with calcofluor white (Fluka) and analyzed with an epifluorescence microscope. The Image J software [49] was used for image analysis.

\section{7. $\beta$-Galactosidase Activity Assay}

The basA promoter was cloned upstream of the lacZ reporter gene in plasmid pMP220::Pbas $A$ (carrying the tetracycline-resistance $\left(\mathrm{Tc}^{\mathrm{R}}\right)$ determinant) as previously described [26]. For reporter gene activity measurements, A. baumannii ATCC $17978\left(\mathrm{Tc}^{\mathrm{S}}\right)$ was transformed with $\mathrm{Pbas} A: \because \operatorname{lacZ}\left(\mathrm{Tc}^{\mathrm{R}}\right)$ and grown for $16 \mathrm{~h}$ at $37^{\circ} \mathrm{C}$ in $\mathrm{M} 9$ medium supplemented with $10 \mu \mathrm{g} / \mathrm{mL}$ Tc. Cultures were then appropriately diluted in TSBD, M9 and M9 supplemented with $100 \mu \mathrm{M}$ DIP, with or without $100 \mu \mathrm{M} \mathrm{FeCl}_{3}$ to reach an initial cell concentration corresponding to $\mathrm{OD}_{600} \sim 0.01$ and incubated at $37{ }^{\circ} \mathrm{C}$ with vigorous shaking. The $\beta$-galactosidase (LacZ) activity expressed by A. baumannii ATCC 17978 (pMP220::PbasA) after 24 and $48 \mathrm{~h}$ growth was determined spectrophotometrically on toluene/SDS-permeabilized cells using $o$-nitrophenyl- $\beta$-D-galactopyranoside as the substrate, and expressed in Miller units [50].

$$
\text { Miller units }=1,000 \times\left[\mathrm{OD}_{420}-\left(1.75 \times \mathrm{OD}_{550}\right)\right] / \text { Volume }(\mathrm{ml}) \times \text { Time }(\mathrm{min}) \times \mathrm{OD}_{600}
$$

\section{Conclusions}

The formation and maturation of $A$. baumannii biofilms depend on the complex interplay of many environmental and cell-associated factors $[13,14]$. In this study, attention has been focused on the role of iron, since this metal is essential for bacterial nutrition and virulence [22-24], and plays a central 
role in host defense from bacterial infection [20]. In agreement with a previous study [25], we showed that planktonic $A$. baumannii cells can overcome iron restriction imposed by a variety of exogenous chelators, likely due to the presence in this species of multiple iron scavenging systems [21,22]. Then, we observed relevant differences in biofilm levels depending on A. baumannii strain and growth medium, and established suitable conditions for testing the effect of iron on biofilm formation. The two most relevant findings of these experiments were: $(i)$ the strong influence of medium composition on biofilm yields; (ii) the high variability in biofilm levels produced by A. baumannii strains of clinical and veterinary origin, irrespective of their genetic relatedness or epidemic potential; (iii) the strain-dependent response of $A$. baumannii biofilms to iron scarcity. Since biofilm formation was either stimulated by iron or not responsive to this metal in the majority of strains tested, we conclude that iron starvation is not sensed as an overall biofilm-inducing stimulus by $A$. baumannii. Consistent with these findings, a recently developed clinical chelator, endowed with extremely high affinity for iron, showed no significant anti-biofilm activity in A. baumannii. Thus, while iron metabolism continues to represent a promising target for $A$. baumannii inhibition, the impressive iron withholding capacity of this bacterium should be taken into account for future development of chelation-based antimicrobial and anti-biofilm therapies.

\section{Supplementary Materials}

Supplementary materials can be accessed at: http://www.mdpi.com/2076-0817/3/3/704/s1 .

\section{Acknowledgments}

This work was supported by a grant from the Italian Ministry of University and Research-PRIN 2012 (prot. 2012WJSX8K) to Paolo Visca. The Authors wish to thank Novartis Pharma AG (Basel, Switzerland) for the generous gift of deferasirox, and the many European laboratories that provided A. baumannii strains.

\section{Author Contributions}

Conceived and designed the experiments: Emanuela Frangipani, Paolo Visca. Supervised the laboratory work: Emanuela Frangipani, Paolo Visca. Performed the experimental work: Valentina Gentile, Carlo Bonchi, Federica Runci, Fabrizia Minandri. Wrote the manuscript: Paolo Visca.

\section{Conflicts of Interest}

The authors declare no conflict of interest.

\section{References}

1. Antunes, L.C.; Visca, P.; Towner, K.J. Acinetobacter baumannii: Evolution of a global pathogen. Pathog. Dis. 2014, 71, 292-301.

2. Falagas, M.E.; Bliziotis, I.A.; Siempos, I.I. Attributable mortality of Acinetobacter baumannii infections in critically ill patients: A systematic review of matched cohort and case-control studies. Crit. Care 2006, 10, R48. 
3. Dijkshoorn, L.; Nemec, A.; Seifert, H. An increasing threat in hospitals: Multidrug-resistant Acinetobacter baumannii. Nat. Rev. Microbiol. 2007, 5, 939-951.

4. Eveillard, M.; Kempf, M.; Belmonte, O.; Pailhoriès, H.; Joly-Guillou, M.L. Reservoirs of Acinetobacter baumannii outside the hospital and potential involvement in emerging human community-acquired infections. Int. J. Infect. Dis. 2013, 17, e802-e805.

5. Endimiani, A.; Hujer, K.M.; Hujer, A.M.; Bertschy, I.; Rossano, A.; Koch, C.; Gerber, V.; Francey, T.; Bonomo, R.A.; Perreten, V. Acinetobacter baumannii isolates from pets and horses in Switzerland: Molecular characterization and clinical data. J. Antimicrob. Chemother. 2011, 66, 2248-2254.

6. Zarrilli, R.; Pournaras, S.; Giannouli, M.; Tsakris, A. Global evolution of multidrug-resistant Acinetobacter baumannii clonal lineages. Int. J. Antimicrob. Agents 2013, 41, 11-19.

7. Durante-Mangoni, E.; Zarrilli R. Global spread of drug-resistant Acinetobacter baumannii: Molecular epidemiology and management of antimicrobial resistance. Future Microbiol. 2011, 6, 407-422.

8. Neonakis, I.K.; Spandidos, D.A.; Petinaki, E. Confronting multidrug-resistant Acinetobacter baumannii: A review. Int. J. Antimicrob. Agents 2011, 37, 102-109.

9. García-Quintanilla, M.; Pulido, M.R.; López-Rojas, R.; Pachón, J.; McConnell, M.J. Emerging therapies for multidrug resistant Acinetobacter baumannii. Trends Microbiol. 2013, 21, 157-163.

10. Jawad, A.; Seifert, H.; Snelling, A.M.; Heritage, J.; Hawkey, P.M. Survival of Acinetobacter baumannii on dry surfaces: Comparison of outbreak and sporadic isolates. J. Clin. Microbiol. 1998, 36, 1938-1941.

11. Antunes, L.C.; Imperi, F.; Carattoli, A.; Visca, P. Deciphering the multifactorial nature of Acinetobacter baumannii pathogenicity. PLoS One 2011, 6, e22674.

12. Giannouli, M.; Antunes, L.C.; Marchetti, V.; Triassi, M.; Visca, P.; Zarrilli, R. Virulence-related traits of epidemic Acinetobacter baumannii strains belonging to the international clonal lineages I-III and to the emerging genotypes ST25 and ST78. BMC Infect. Dis. 2013, 13, 282.

13. Gaddy, J.A.; Actis, L.A. Regulation of Acinetobacter baumannii biofilm formation. Future Microbiol. 2009, 4, 273-278.

14. Longo, F.; Vuotto, C.; Donelli, G. Biofilm formation in Acinetobacter baumannii. New Microbiol. 2014, 37, 119-127.

15. Rodríguez-Baño, J.; Martí, S.; Soto, S.; Fernández-Cuenca, F.; Cisneros, J.M.; Pachón, J.; Pascual, A.; Martínez-Martínez, L.; McQueary, C.; Actis, L.A.; et al. Spanish Group for the Study of Nosocomial Infections (GEIH). Biofilm formation in Acinetobacter baumannii: Associated features and clinical implications. Clin. Microbiol. Infect. 2008, 14, 276-278.

16. Lee, H.W.; Koh, Y.M.; Kim, J.; Lee, J.C.; Lee, Y.C.; Seol, S.Y.; Cho, D.T.; Kim, J. Capacity of multidrug-resistant clinical isolates of Acinetobacter baumannii to form biofilm and adhere to epithelial cell surfaces. Clin. Microbiol. Infect. 2008, 14, 49-54.

17. Wroblewska, M.M.; Sawicka-Grzelak, A.; Marchel, H.; Luczak, M.; Sivan, A. Biofilm production by clinical strains of Acinetobacter baumannii isolated from patients hospitalized in two tertiary care hospitals. FEMS Immunol. Med. Microbiol. 2008, 53, 140-144.

18. McQueary, C.N.; Actis, L.A. Acinetobacter baumannii biofilms: Variations among strains and correlations with other cell properties. J. Microbiol. 2011, 49, 243-250. 
19. Tomaras, A.P.; Dorsey, C.W.; Edelmann, R.E.; Actis, L.A. Attachment to and biofilm formation on abiotic surfaces by Acinetobacter baumannii: Involvement of a novel chaperone-usher pili assembly system. Microbiology 2003, 149, 3473-3484.

20. Weinberg, E.D. Iron availability and infection. Biochim. Biophys. Acta 2009, 1790, 600-605.

21. Antunes, L.C.; Imperi, F.; Towner, K.J.; Visca, P. Genome-assisted identification of putative iron-utilization genes in Acinetobacter baumannii and their distribution among a genotypically diverse collection of clinical isolates. Res. Microbiol. 2011, 162, 279-284.

22. Zimbler, D.L.; Penwell, W.F.; Gaddy, J.A.; Menke, S.M.; Tomaras, A.P.; Connerly, P.L.; Actis, L.A. Iron acquisition functions expressed by the human pathogen Acinetobacter baumannii. Biometals 2009, 22, 23-32.

23. Gaddy, J.A.; Arivett, B.A.; McConnell, M.J.; López-Rojas, R.; Pachón, J.; Actis, L.A. Role of acinetobactin-mediated iron acquisition functions in the interaction of Acinetobacter baumannii strain ATCC $19606^{\mathrm{T}}$ with human lung epithelial cells, Galleria mellonella caterpillars, and mice. Infect. Immun. 2012, 80, 1015-1024.

24. Mortensen, B.L.; Skaar, E.P. The contribution of nutrient metal acquisition and metabolism to Acinetobacter baumannii survival within the host. Front. Cell. Infect. Microbiol. 2013, 3, 95.

25. De Léséleuc, L.; Harris, G.; KuoLee, R.; Chen, W. In vitro and in vivo biological activities of iron chelators and gallium nitrate against Acinetobacter baumannii. Antimicrob. Agents Chemother. 2012, 56, 5397-5400.

26. Antunes, L.C.; Imperi, F.; Minandri, F.; Visca, P. In vitro and in vivo antimicrobial activities of gallium nitrate against multidrug-resistant Acinetobacter baumannii. Antimicrob. Agents Chemother. 2012, 56, 5961-5970.

27. Minandri, F.; Bonchi, C.; Frangipani, E.; Imperi, F.; Visca, P. Promises and failures of gallium as an antibacterial agent. Future Microbiol. 2014, 9, 379-397.

28. Nucleo, E.; Steffanoni, L.; Fugazza, G.; Migliavacca, R.; Giacobone, E.; Navarra, A.; Pagani, L.; Landini, P. Growth in glucose-based medium and exposure to subinhibitory concentrations of imipenem induce biofilm formation in a multidrug-resistant clinical isolate of Acinetobacter baumannii. BMC Microbiol. 2009, 9, 270.

29. Smith, M.G.; Gianoulis, T.A.; Pukatzki, S.; Mekalanos, J.J.; Ornston, L.N.; Gerstein, M.; Snyder, M. New insights into Acinetobacter baumannii pathogenesis revealed by high-density pyrosequencing and transposon mutagenesis. Genes Dev. 2007, 21, 601-614.

30. Guterman, S.K. Colicin B: Mode of action and inhibition by enterochelin. J. Bacteriol. 1973, 114, 1217-1224.

31. Fournier, P.E.; Vallenet, D.; Barbe, V.; Audic, S.; Ogata, H.; Poirel, L.; Richet, H.; Robert, C.; Mangenot, S.; Abergel, C.; et al. Comparative genomics of multidrug resistance in Acinetobacter baumannii. PLoS Genet. 2006, 2 , e7.

32. Iacono, M.; Villa, L.; Fortini, D.; Bordoni, R.; Imperi, F.; Bonnal, R.J.; Sicheritz-Ponten, T.; de Bellis, G.; Visca, P.; Cassone, A.; et al. Whole-genome pyrosequencing of an epidemic multidrug-resistant Acinetobacter baumannii strain belonging to the European clone II group. Antimicrob. Agents Chemother. 2008, 52, 2616-2625. 
33. D’Arezzo, S.; Principe, L.; Capone, A.; Petrosillo, N.; Petrucca, A.; Visca, P. Changing carbapenemase gene pattern in an epidemic multidrug-resistant Acinetobacter baumannii lineage causing multiple outbreaks in central Italy. J. Antimicrob. Chemother. 2011, 66, 54-61.

34. Van Dessel, H.; Dijkshoorn, L.; van der Reijden, T.; Bakker, N.; Paauw, A.; van den Broek, P.; Verhoef, J.; Brisse, S. Identification of a new geographically widespread multiresistant Acinetobacter baumannii clone from European hospitals. Res. Microbiol. 2004, 155, 105-112.

35. Ohman, D.; Sadoff, J.; Iglewski, B. Toxin A-deficient mutants of Pseudomonas aeruginosa PA103: Isolation and characterization. Infect. Immun. 1980, 28, 899-908.

36. O’Toole, G.; Kaplan, H.B.; Kolter, R. Biofilm formation as microbial development. Annu. Rev. Microbiol. 2000, 54, 49-79.

37. Jurcisek, J.A.; Dickson, A.C.; Bruggeman, M.E.; Bakaletz, L.O. In vitro biofilm formation in an 8-well chamber slide. J. Vis. Exp. 2011, 47, doi:10.3791/2481.

38. Sigma-Aldrich. Available online: www.sigmaaldrich.com (accessed on 14 August 2014).

39. Hider, R.; Xiaole, K.; Lucker, T.; Conlon, K.; Harland, R. SPD602 is a selective iron chelator which is able to mobilise the non-transferrin-bound iron pool. Blood 2013, 122, 1673.

40. Luo, G.; Spellberg, B.; Gebremariam, T.; Lee, H.; Xiong, Y.Q.; French, S.W.; Bayer, A.; Ibrahim, A.S. Combination therapy with iron chelation and vancomycin in treating murine staphylococcemia. Eur. J. Clin. Microbiol. Infect. Dis. 2014, 33, 845-851.

41. Lewis, R.E.; Pongas, G.N.; Albert, N.; Ben-Ami, R.; Walsh, T.J.; Kontoyiannis, D.P. Activity of deferasirox in Mucorales: Influences of species and exogenous iron. Antimicrob. Agents. Chemother. 2011, 55, 411-413.

42. Nisbet-Brown, E.; Olivieri, N.F.; Giardina, P.J.; Grady, R.W.; Neufeld, E.J.; Séchaud, R.; Krebs-Brown, A.J.; Anderson, J.R.; Alberti, D.; Sizer, K.C.; et al. Effectiveness and safety of ICL670 in iron-loaded patients with thalassaemia: A randomised, double-blind, placebo-controlled, dose-escalation trial. Lancet 2003, 361, 1597-1602.

43. Vallenet, D.; Nordmann, P.; Barbe, V.; Poirel, L.; Mangenot, S.; Bataille, E.; Dossat, C.; Gas, S.; Kreimeyer, A.; Lenoble, P.; et al. Comparative analysis of Acinetobacters: Three genomes for three lifestyles. PLoS One 2008, 19, e1805.

44. D’Andrea, M.M.; Giani, T.; D’Arezzo, S.; Capone, A.; Petrosillo, N.; Visca, P.; Luzzaro, F.; Rossolini, G.M. Characterization of pABVA01, a plasmid encoding the OXA-24 carbapenemase from Italian isolates of Acinetobacter baumannii. Antimicrob. Agents Chemother. 2009, 53, 3528-3533.

45. Vaneechoutte, M.; Devriese, L.A.; Dijkshoorn, L.; Lamote, B.; Deprez, P.; Verschraegen, G.; Haesebrouck, F. Acinetobacter baumannii-infected vascular catheters collected from horses in an equine clinic. J. Clin. Microbiol. 2000, 38, 4280-4281.

46. Zordan, S.; Prenger-Berninghoff, E.; Weiss, R.; van der Reijden, T.; van den Broek, P.; Baljer, G.; Dijkshoorn, L. Multidrug-resistant Acinetobacter baumannii in veterinary clinics, Germany. Emerg. Infect. Dis. 2011, 17, 1751-1754.

47. MacKenzie, F.M.; Struelens, M.J.; Towner, K.J.; Gould, I.M.; ARPAC Steering Group; ARPAC Consensus Conference Participants. Report of the Consensus Conference on Antibiotic Resistance; Prevention and Control (ARPAC). Clin. Microbiol. Infect. 2005, 11, 938-954. 
48. Towner, K.J.; Levi, K.; Vlassiadi, M.; on behalf of the ARPAC Steering Group. Genetic diversity of carbapenem-resistant isolates of Acinetobacter baumannii in Europe. Clin. Microbiol. Infect. 2008, 14, 161-167.

49. ImageJ. Available online: http://rsbweb.nih.gov/ij/ (accessed on 14 August 2014).

50. Miller, J.H. Experiments in Molecular Genetics; Cold Spring Harbor Laboratory: Cold Spring Harbor, NY, USA, 1972; pp. 252-255.

(C) 2014 by the authors; licensee MDPI, Basel, Switzerland. This article is an open access article distributed under the terms and conditions of the Creative Commons Attribution license (http://creativecommons.org/licenses/by/3.0/). 